Methods A General Paediatric telephone triage was introduced, operating for 12 hours each day $(9 \mathrm{am}-9 \mathrm{pm}) 7$ days per week. This service was staffed by senior paediatric clinicians and received calls from GP surgeries, NHS24 and COVID assessment centres. The clinician delivering the service discussed all acute referrals with referring clinician and was able to offer immediate advice with regard to most appropriate management. Data from each acute referral received including the outcome of the call was collected prospectively.

Results Data has been collected for 2834 acute referrals to our Paediatric Telephone Triage service from its establishment in March 2020. Of these calls, 57\% (1627) had been seen in a face-to-face consultation with their GP prior to referral.

We found that following telephone triage with referring clinician acute attendance and assessment was not required in $972 / 2834(34 \%)$ cases. In $447 / 2834(16 \%)$ cases it was felt that the child did not require assessment in secondary care at all and the GP was provided with advice in relation to ongoing management. In 128/2834 (4.5\%) referrals the child was streamed into General Paediatric clinic (including utilisation of rapid access and urgent telephone consultations). The remaining calls resulted in patients being seen in primary care facilities (including COVID assessment centres) or being streamed to other specialities within the hospital.

Conclusions Implementing a telephone-based triage service for paediatrics has reduced unnecessary unscheduled care attendances. Primary Care access to a senior paediatric clinician by telephone has had a positive impact on the number of children being referred for acute assessment. Utilisation of Rapid Access Clinics and urgent Telephone Consultations can help ensure that children are seen in a timely fashion and help reduce pressure on front door services.

\section{DIAGNOSIS OF UNILATERAL CEREBELLAR HYPOPLASIA IN 10 YEAR OLD CHILD FOLLOWING REFERRAL FOR AUTISM SPECTRUM DISORDER ASSESSMENT}

Catherine Bushill, Jessica Slater. UK

\subsection{6/bmjpo-2021-RCPCH.137}

Background A 10 year old only child of Polish parents was referred via the concerning behaviours pathway to assess her for developmental impairment. Her medical history was complex, beginning with being born prematurely at $23+4$ weeks of gestation. Despite follow up until the age of 5 years she did not meet criteria for further investigation or follow up at the time. Investigations by community paediatrics revealed significant organic neurological abnormalities as well as important psycho-social issues, culminating in a diagnosis of both Attention Deficit Hyperactivity Disorder (ADHD) and Autism Spectrum Disorder (ASD).

Objectives Interesting case highlighting important aspects of Community Paediatrics.

Methods The child was seen by the community paediatrician, who listened to concerns of her parents, looked back in depth at the patient's history and referred onto the relevant teams for assessment of her needs.

Results The community paediatrician was an advocate for the patient and parents in obtaining the support she needed for her education and development. When requests were denied for an Education, Health and Care Plan (EHCP), and a speech and language assessment referral rejected due to the child being bilingual, the community paediatrician was able to work alongside a Special Educational Needs and Disabilities (SEND) advocate to access these for the patient.

Despite support from her parents, she had struggled in mainstream school - not only with impairment in gross and fine motor skills, but also with cognition. She had low selfesteem and found making friends hard. Due to the Covid19 pandemic she had home-schooling and the combination of increased supervision, working at her own pace and reduced pressure on social skills she had been thriving. However the pandemic has delayed assessment by speech and language, occupational and physical therapy.

Following discussion of parental concerns regarding cerebral palsy, and having a grossly normal neurological examination, an MRI was requested which showed almost complete absence of the left cerebellar hemisphere thought to be as a result of perinatal insult. Armed with MRI results the child was found to have an abnormal cerebellar examination.

Conclusions This important case highlights the benefits of a multi-disciplinary team working closely with school and family. This case demonstrates the central role of the community paediatrician in providing holistic care of patients and how they are well placed to advocate for such patients and their families. This patient was referred for ASD assessment, but for this patient ADHD and ASD are part of her picture, but not the only concern.

Importance of investigating based on new assessment is highlighted. Despite MRI previously being felt unnecessary, it revealed important findings which will help to more thoroughly understand her needs. Previous assessment may lack vital information or the patient's presentation may have evolved.

This case also illustrates possible health inequalities for patients for whom English is not their first language. Language barriers are associated with inequalities in access to and quality of healthcare delivered and may have contributed to the late diagnosis of cerebellar hypoplasia. It would be interesting to see if cultural differences could also have had an impact.

\section{POST COVID-19 LOCKDOWN 1.0 RAPID HEALTH SERVICES EQUITY ASSESSMENT FOR CHILDREN AND YOUNG PEOPLE WITH SPECIAL EDUCATIONAL NEEDS AND DISABILITIES}

Caroline Gribbon, Michael Alexander, Michelle Heys, Angela Bartley, Liam Crosby. UK

\subsection{6/bmjpo-2021-RCPCH.138}

Background The COVID-19 Pandemic has amplified the preexisting health and social inequalities in our society. The impact of the pandemic, from both direct and indirect consequences, on children and young people (CYP) with special educational needs and disability (SEND) is likely to be huge.

Objectives In partnership with an inner-city London council we conducted a rapid health services equity assessment with the aim of understanding the impact of the COVID-19 pandemic and associated first lockdown (23/03-01/06/20) on CYP with SEND and their families attending our health services. We sought to understand how their mental and physical health, accessing services, education, social connectedness and household financial security had changed as a result of the pandemic. The findings would help us understand the broader 
Abstract 246 Table 1 Describes change in key health measures. $40 \%$ of CYP and $43 \%$ of parents reported a deterioration in mental health but only 6 families accessed emotional support groups

\begin{tabular}{lllll}
\hline & Better & Same & Worse & n/a \\
\hline CYP Mental Health & $13 \%$ & $47 \%$ & $40 \%$ & \\
Parent Mental Health & $8 \%$ & $49 \%$ & $43 \%$ & \\
CYP Physical Health & $19 \%$ & $48 \%$ & $33 \%$ & \\
Parent Physical Health & $9 \%$ & $59 \%$ & $32 \%$ & \\
CYP exercise & $8 \%$ & $33 \%$ & $56 \%$ & $3 \%$ \\
CYP sleep & $7 \%$ & $50 \%$ & $43 \%$ & \\
Finance & $4 \%$ & $55 \%$ & $41 \%$ & \\
Housing & $4 \%$ & $83 \%$ & $13 \%$ & \\
Employment & $0 \%$ & $63 \%$ & $37 \%$ & \\
\hline
\end{tabular}

social and health needs of this population and shape our future service.

Methods A questionnaire was designed and piloted with two parents. The sample was non-random/convenience sampling taken from a contact list of CYP with SEND who were shielding or considered vulnerable. Participants were informed this was part of a service evaluation and consented to be interviewed. A total of 75 telephone interviews were conducted. The council completed 46 questionnaires and in October 2020 the medical team attempted contact with a further 94 families. 46 families answered the telephone and 29 agreed to complete the questionnaire. Ethics was not sought as it was a health service evaluation.

Results The participants children were between 0-19 years with the majority $(40 \%)$ between 5-9 years. $89 \%$ of the sample was BAME. 55\% of the school-age children were eligible for a school place during lockdown however only 21\% of these opted to attend school. Parents cited reasons for not attending school as shielding, fear of COVID-19 and lack of equipment.

The most common services accessed were primary care, specialist services (eg child development centre) and food banks. Of the 27 families describing worse employment situation, only 1 accessed the jobcentre. Of the 31 families with worse financial situation 15 accessed the food bank and 1 accessed the Citizens' Advice Bureau.

Conclusions Further exploration is required with validated measures to understand the impact of the pandemic and of associated interventions (eg lockdown) on health and mental health in particular. Our survey shows that emotional and financial services were not widely used despite the difficulties many faced. Signposting families to these services is being prioritised in our clinical interactions and through development of a social prescribing model. More in-depth qualitative research is planned on this population exploring the interplay between social determinants and COVID in CYP with SEND and their families.

\section{VALIDITY OF AN IVH AND PVL PREVENTION BUNDLE INTERVENTIONS IN A CENTRE WITH A VERY LOW INCIDENCE OF SEVERE IVH: A CASE-CONTROL STUDY}

Mavis Xuanru Tan, Abdul Alim Abdul Haium, Nirmal Kavalloor Visruthan, Victor Samuel Rajadurai, Thowfique Ibrahim. Singapore
Background Severe intraventricular haemorrhage (IVH, Papile's Grades 3 and 4) can cause significant neurodevelopmental impairment in $43 \%$ of affected infants.

Objectives The objectives of this study were to study the risk factors for severe IVH and evaluate the validity of an IVH prevention bundle in VLBW infants in a tertiary care centre with very low incidence of severe IVH.

Methods All VLBW infants born between January 2013 to December 2018 were included in the study. Data was collected from a prospectively maintained database and retrospective chart review. For each severe IVH infant (study group), 2 IVH-free infants were identified as control and were closely matched for birth weight $( \pm 100 \mathrm{~g})$ and gestational age $( \pm 1$ week). Eighteen risk factors studied under severe IVH review document and six risk factors explored under interventions were combined, edited for overlaps or repeats and compared between the two groups. Bundle of interventions to reduce severe IVH included 1) Neutral head position and developmental care (2012); 2) Optimising the time of administration of antenatal steroid to mothers at risk of preterm birth at $\leq 24$ weeks gestation by administering the drug at least 48 hours before the delivery (2014); 3) Antenatal magnesium sulphate administration for neuroprotection to mothers in preterm labour with foetus of $\leq 32$ weeks gestation (2015); 4) Protocol for prevention of hypothermia in newborn infants (2016); 5) Patent ductus arteriosus (PDA) management protocol featuring early selective treatment of high-risk infants (2016); and 6) Prophylactic indomethacin for IVH prevention guideline (2016, modified in 2017). Univariate and multivariate analysis were conducted.

The bundle was defined as valid, if there was a statistically significant difference in incidence of corresponding risk factors between the 2 groups and $\geq 50 \%$ reduction in incidence of severe IVH in the post-intervention period. The stratified yearly incidences of IVH were captured.

Results 1261 VLBW infants were born during the study period and $48(3.8 \%)$ infants developed severe IVH with 96 matched infants identified as controls. Baseline maternal and infant characteristics were comparable. The incidence of severe IVH was $6.2 \%$ and $1.9 \%$ in the 2012 and 2018 cohorts respectively. On univariate analysis, maternal tocolytic therapy, difficult resuscitation at birth, difficult procedures, hypercapnia, high-frequency oscillatory ventilation and, multiple inotropes for hypotension during the first week of life were found to be statistically significant. Multivariate analysis revealed tocolytic therapy (OR $0.26,95 \% \mathrm{CI}, 0.10-0.66$ ) to be an independent protective factor whereas delivery room intubation (OR 8.77, 95\% CI, 1.10 - 70.06) and multiple inotropes (OR 2.32, 95\% CI, 1.44 - 3.76) were significant risk factors for severe IVH.

Conclusions The IVH prevention bundle interventions were found to be valid and the bundle model can be replicated to reduce the overall incidence of severe IVH in VLBW infants to less than $2 \%$.

\section{DOES THE 'CRIB II' SCORING SYSTEM SCORE WELL IN MORTALITY RISK PREDICTION IN INDIA?}

Amruta Phatak, Abhimanyu Niswade, Rajkumar Meshram, Swapnil Wathore. India

10.1136/bmjpo-2021-RCPCH.140

Background Introduction: 